\title{
REMARKS ON COSMOLOGY
}

\author{
G. C. MC VITTIE \\ University of Illinois Observatory, Urbana, Illinois, U.S.A.
}

First, I should like to say something about the use of highly specialized models in cosmology. The Einstein-de Sitter model is a relativistic model in which the cosmical constant and the space-curvature constant are both equated to zero. Likewise, the pressure is assumed to be zero throughout the history of the universe, except perhaps at the initial instant. It is well-known that the first two constants can be determined from observation, if not at present, at any rate as the data are refined in the future. Hence, I think it is a weakness to prejudge the issue and assign a priori values. Nor is it self-evident to me that the pressure must always have been zero even if it is zero now. Composite models, with nonzero pressure at first, followed by a zero-pressure condition, need to be examined.

The steady-state model is equally specialized. It assumes that the Hubble "constant" is indeed a constant of nature independent of the time; it also assigns zero value to the space curvature. Further, one must assume a very special relation between the initial value of the Hubble constant and the creation-of-matter vector. If this relation is not satisfied, a nonsteady-state universe results.

Difficulties arise when such too-specialized models are used; for example, a very high average density of matter. The law for changing the angular diameter of a source of constant area found by Hoyle in the Einstein-de Sitter universe cannot hold for all relativistic models, as the following argument shows: the effect is not present in the steady-state model, as he has told us. The steady-state model is identical with the de Sitter universe of general relativity, at least as far as the motion of light is concerned, which is the only relevant consideration in this connection. Therefore, the effect is absent in one of the relativistic models, and in my opinion it would also be absent in all those for which the expansion factor was appropriately chosen.

In my view of cosmology, observation enables us to reject certain classes of models but it does not permit us to pinpoint some particular model among those that remain. For example, consider the red-shift data on clusters of galaxies published by Humason, Mayall, and Sandage [1]. Sandage's analysis of these data gave a negative acceleration for the expansion; my own later analysis of these same data [2], using a somewhat different method, confirmed his result. I think that this conclusion should stand until more measurements of red-shifts and apparent magnitudes of galaxies in clusters show that we must alter it. The negative acceleration rules out a large class of relativistic models, and unfortunately for the supporters of the steady-state theory, it rules out that model also.

Let me now illustrate to what extent the general relativistic model universe 
can be used to interpret the counts of extragalactic radio sources. I take the data of Mills and Slee [3] purely as illustrative. The empirical relation (allowing for the red-shift effect) of numbers as a function of decreasing flux density can be interpreted in three ways: (1) All sources radiate constantly in time and they are of two kinds, viz. NGC 1275 sources and Cygnus A sources. Then a satisfactory fit is found if there are about 4000 sources like NGC 1275 to one of Cygnus A type. (2) All sources are of Cygnus A type, but are subject to large secular variations of intrinsic power output. Even the nearest ones must have been radiating, at the instant the radiation by which they are now observed left them, at least three times stronger than Cygnus A. (3) All sources are of Cygnus A type, but there are no secular changes of radiative power. In this case, no fit at all can be found, which illustrates the point that Mills's data, his view to the contrary, can indeed be used to rule out certain possibilities though they do not suffice to make a unique selection among the remainder.*

Specialized models unfortunately have to be used in cases in which total effect from all sources in the universe is in question, because integrations " to infinity" must now be performed and this can be done only for specific models. This means that if we want to go to all distances the exact law of velocity and acceleration must be given a priori and so must the character of the space curvature. At present, neither of these items can be deduced from observation with the required degree of certainty. A problem of this kind is that of the general isotropic background radiation, which I will take as being of $500^{\circ} \mathrm{K}$ at $100 \mathrm{Mc} / \mathrm{s}$. My colleague, Stanley P. Wyatt, Jr., and I have recently been working out this effect for a Milne universe. Our choice was dictated by the fact that in this model the effect of the motion of recession is alone relevant, and the space curvature is hyperbolic. Gravitational effects are neglected in this universe. The results are these: if a Milne universe is filled with $M$ 31-type galaxies, the background radiation corresponds to six such galaxies per cubic megaparsec, but if it is filled with Cygnus A-type galaxies, then there are $10^{-0}$. We have also shown that the contribution to the background radiation, owing to those extremely remote galaxies whose optical radiation is lengthened to $100 \mathrm{Mc} / \mathrm{s}$ by the red-shift, is quite negligible. It is indeed down by a factor of $10^{-6}$ as compared to the nonthermal emission from the, relatively speaking, nearer galaxies. Such investigations are useful only as indicators of what can be expected; many models possessing extreme properties in one direction or another must be used, from which we can expect to obtain upper and lower limits of what might be observed.

\section{REFERENCES}

[1] Humason, M. L., Mayall, N. U., and Sandage, A. R. A.J. 61, 97, 1956.

[2] McVittie, G. C. Handbuch der Physik, Vol. 53. (In press.)

[3] Mills, B. Y., and Slee, O. B. Aust. J. Phys. 10, 162, 1957.

[4] McVittie, G. C. Aust. J. Phys. 10, 331, 1957.

* Details will be found in [4]. The curves I and II of Fig. 2 were shown on the screen. Curve I refers to (1) and (2) above, curve II to (3). 


\section{Discussion}

Gold: The radio source counts were taken to represent cosmological information when the gradient of the $\log N$ vs. $\log I$ plot appeared to be much more than $3 / 2$. This, together with isotropy, could not result from anything except a variation of density with range. Since this is known to be an effect in the optically surveyed parts of the universe, it was argued that it must also result from farther away, and that therefore a great many sources had to be far away. If now the number count results give in fact the $3 / 2$ gradient, there is no basis for supposing a sufficient fraction of the sources to be so distant. Optically invisible and intrinsically weak radio sources may dilute the data, and there is then no cosmological relevance. It would be necessary to have some indication of distance for a substantial number before using the data for any kind of cosmological considerations.

Ryle: The purpose behind my paper was to show that on the present evidence-whether we use the Cambridge or the Mills results-it seems that we must suppose those sources to be very powerful extragalactic objects whose radio luminosities approach that of Cygnus $A$. Under these circumstances even the present surveys reach to distances where the red-shift is important, and on a steady-state model we should find a marked deficit of faint sources. This has not been observed, and the results of both surveys therefore do not appear to support the steady-state model. Any greater dispersion in the luminosity function would enhance the effect.

Hoyle: Could I ask Ryle what power level he took for his sources?

Ryle: The lower limit we derived for $P$ was above $1 / 30$ that of Cygnus, and this would produce the marked effects reported.

Oort: Was not Gold too pessimistic in his statement that the radio sources are not likely to contribute in the field of cosmology? I think there are reasons to suppose that at least a fraction-if not a large part-of the weaker sources are at distances that are cosmologically interesting. For instance, the small diameter found for some sources indicate that there are sources of the Cygnus A type at very large distances.

With reference to Hoyle's communication I should like to point to the observational evidence for isotropy of the universe and also to the possibility of obtaining some information on the density in the universe by a study of internal motions in the clusters and localized clouds into which almost all the galaxies seem to be concentrated.

Hoyle: I don't think the total density can ever be obtained from dynamical measures, the reason being that a uniform distribution of material does not produce any dynamical effect. Thus, so far as dynamical criteria are concerned one can add a uniform contribution to the total density, and this may possibly be a large term, much larger than the nonuniform component. 